\title{
Middle ear adenoma with neuroendocrine differentiation: relate of two cases and literature review
}

\author{
Aline Gomes Bittencourt', Robinson Koji Tsujp, Francisco Cabral Junior ${ }^{3}$, Larissa Vilela Pereira ${ }^{3}$, Anna Carolina de Oliveira Fonseca', \\ Venâncio Alves ${ }^{4}$, Ricardo Ferreira Bento5.
}

1) Otolaryngologist, Ph.D. Student. Department of Otolaryngology, University of São Paulo School of Medicine, São Paulo, Brazil.

2) M.D., Ph.D. - Associated Doctor. Department of Otolaryngology, University of São Paulo School of Medicine, São Paulo, Brazil.

3) M.D., Otolaryngology Resident. Department of Otolaryngology, University of São Paulo School of Medicine, São Paulo, Brazil

4) M.D. Ph.D. Professor and Chairman - Department of Patology, University of São Paulo School of Medicine, São Paulo, Brazil.

5) M.D. Ph.D. Professor and Chairman - Department of Otolaryngology, University of São Paulo School of Medicine, São Paulo, Brazil.

Institution: Departamento de Otorrinolaringologia, Hospital das Clínicas, Universidade de São Paulo.

São Paulo / SP - Brazil.

Mailing address: Aline Gomes Bittencourt - Departamento de Otorrinolaringologia - Hospital das Clínicas - Universidade de São Paulo - Av. Dr. Enéas de Carvalho Aguiar, 255, 6ªndar/sala 6167 - São Paulo / SP - Brazil / Zip code: 05403-000 - Telephone: (+ 55 11) 3088 0299 - E-mail: alinebittencourt@ hotmail.com Article received on December 20 ${ }^{\text {th }}, 2012$. Article accepted on February 18 ${ }^{\text {th }}, 2013$.

\section{SUMMARY}

Introduction: Adenomas with neuroendocrine differentiation are defined as neuroendocrine neoplasms, and they are rarely found in the head and neck.

Objective: To describe two cases of a middle ear adenoma with neuroendocrine differentiation, with a literature review.

Case Report: Patient 1 was a 41-year-old woman who presented with a 3-year history of left aural fullness associated with ipsilateral "hammer beating" tinnitus. Patient 2 was a 41 -year-old male who presented with unilateral conductive hearing loss. Conclusion: Adenoma with neuroendocrine differentiation of the middle ear is a rare entity, but it should be considered in patients with tinnitus, aural fullness, and a retrotympanic mass and remembered as a diferential diagnosis of tympanic paraganglioma.

Keywords: Neuroendocrine Tumors; Hearing Loss; Adenoma.

\section{INTRODUCTION}

Neuroendocrine cells are generally present in the respiratory epithelium as well as in the bronchial and intestinal mucosa. A few of these cells are also associated with epithelial structures found throughout the body, including the head and neck. Adenomas with neuroendocrine differentiation are defined as neuroendocrine neoplasms, and they are rarely found in the head and neck. When they occur in this region, they are more commonly found in the larynx (1). They usually grow in the lungs or gastrointestinal tract $(1,2)$.

\section{CASE REPORTS}

Patient 1 was a 41-year-old woman who presented with a 3-year history of left aural fullness associated with ipsilateral "hammer beating" tinnitus. Over the following 8 months, the patient developed left-side otalgia and was treated with systemic antibiotics but without any improvement.

The patient consulted a colleague who raised the possibility of a tympanic paraganglioma. Computed tomography (CT) of the temporal bones revealed a material with a soft tissue density that diffusely obliterated the left mastoid cells, but with no signs of ossicular chain erosion (Figure 1). Angiography of the temporal bone showed a lobulated solid lesion with defined limits and contrast uptake within the left middle ear and the epitympanic recess.

The patient consulted our institution when her symptoms persisted. She had no complaints of hearing loss or otorrhea. On examination, the patient was found to be in good general condition and without neurological deficits. Otoscopy of the right ear was normal, but a retrotympanic, non-pulsatile mass was detected in the left ear (Figure 2). Figure 3 demonstrates her puretone audiometry, with a 10dB-gap in the left ear. She subsequently underwent a left radical mastoidectomy with total resection of the lesion. Intraoperatively, the tumor had a fibrous aspect and filled the antrum and the entire tympanic cavity, involving the ossicular chain. The histopathological study was compatible with middle ear adenoma with neuroendocrine differentiation.

Patient 2 was a 41-year-old man who presented with unilateral conductive hearing loss. CT scan showed a middle ear mass in the promontory mimicking a tympanic 

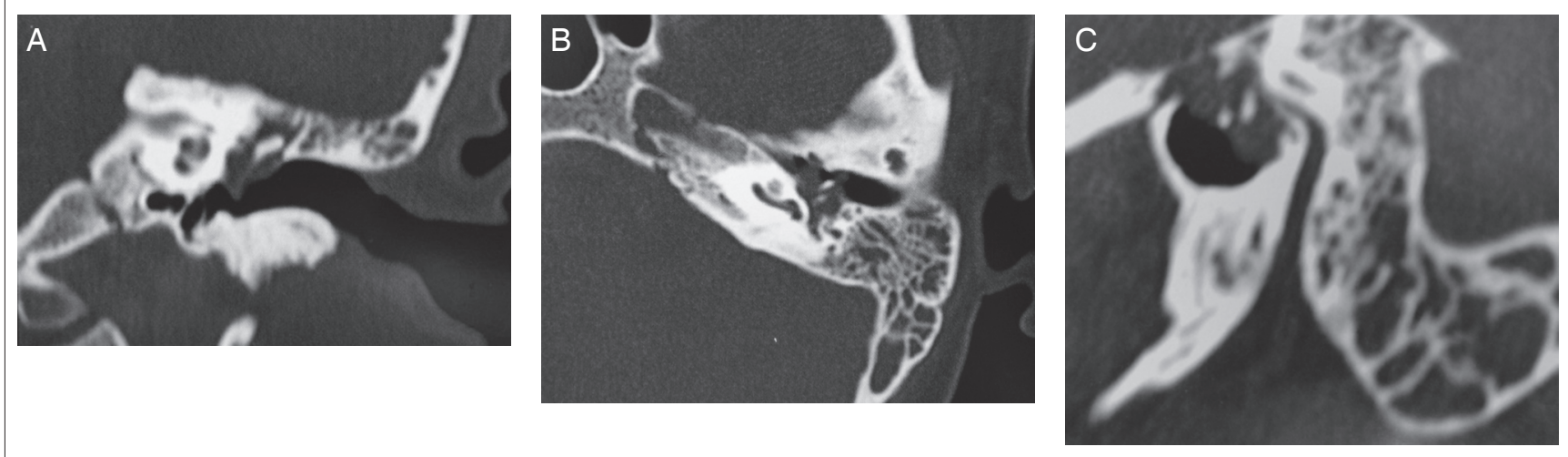

Figure 1. Computed tomography of the temporal bones revealed a material with a soft tissue density that diffusely obliterated the left mastoid cells, but with no signs of ossicular chain erosion. A. Coronal B. Axial C. Transverse

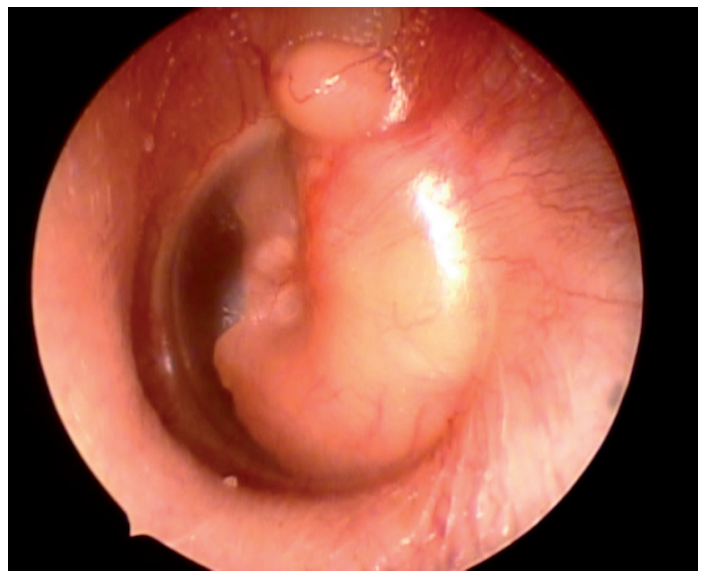

Figure 2. Otoscopy of the left ear demonstrating a retrotympanic non-pulsatile mass.

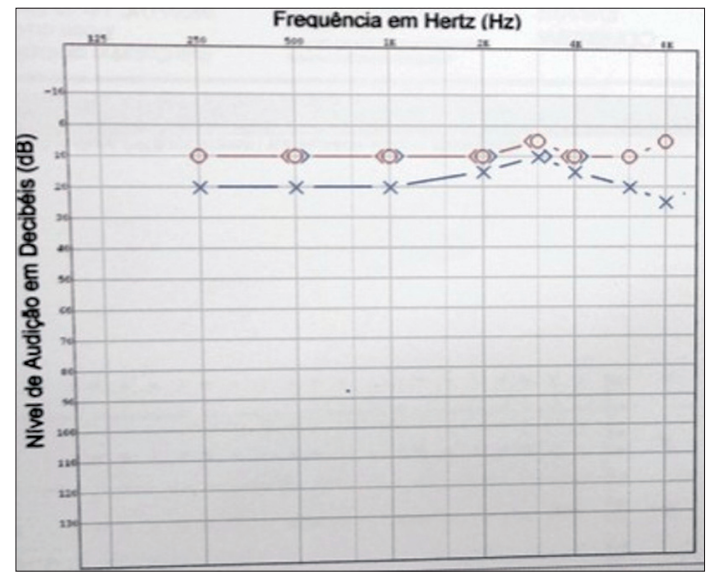

Figure 3. Pure tone audiometry showing a gap in the left ear.

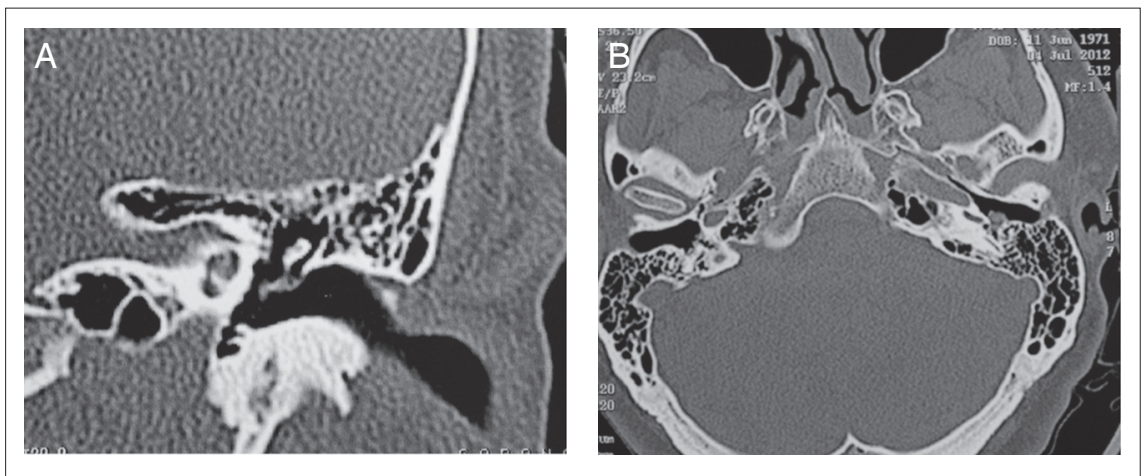

Figure 4. Computed tomography of the temporal bones revealing a small soft tissue density material in the left tympanic cavity. A. Coronal B. Axial

paraganglioma (Figure 4). Magnetic ressonance imaging (MRI) of the temporal bones revealed a small mass in the left tympanic cavity with isosignal in T1 and post-contrast enhancement on T2 sequences(Figure 5). The patient underwent surgical intervention through a supra-meatal approach that completely removed the mass. The tumor was brownish and elastic, involved the ossicles and extended to the promontory. Histology and immunohistochemical reactions were also compatible with middle ear adenoma with neuroendocrine differentiation (Figure 6). 


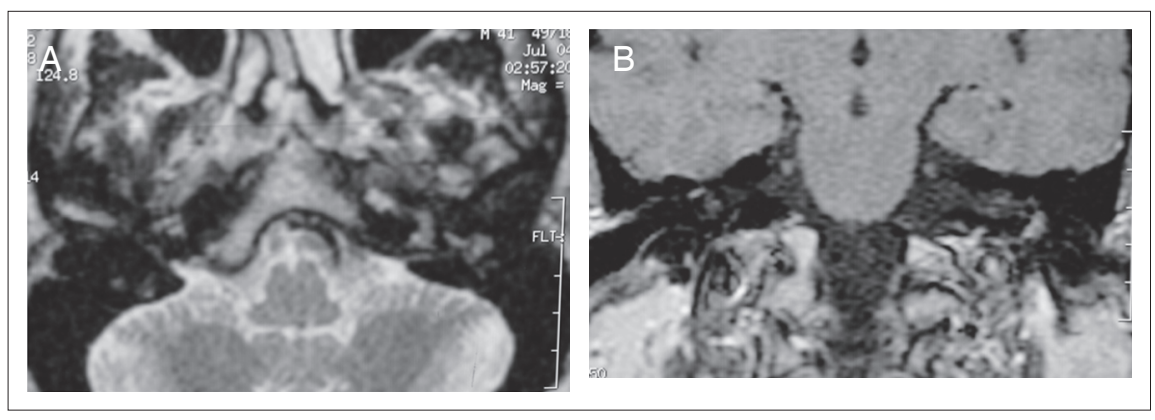

Figure 5. A: MRI of the temporal bones revealing a small mass in the left tympanic cavity with isosignal in T1 (A) and post-contrast enhancement on T2.
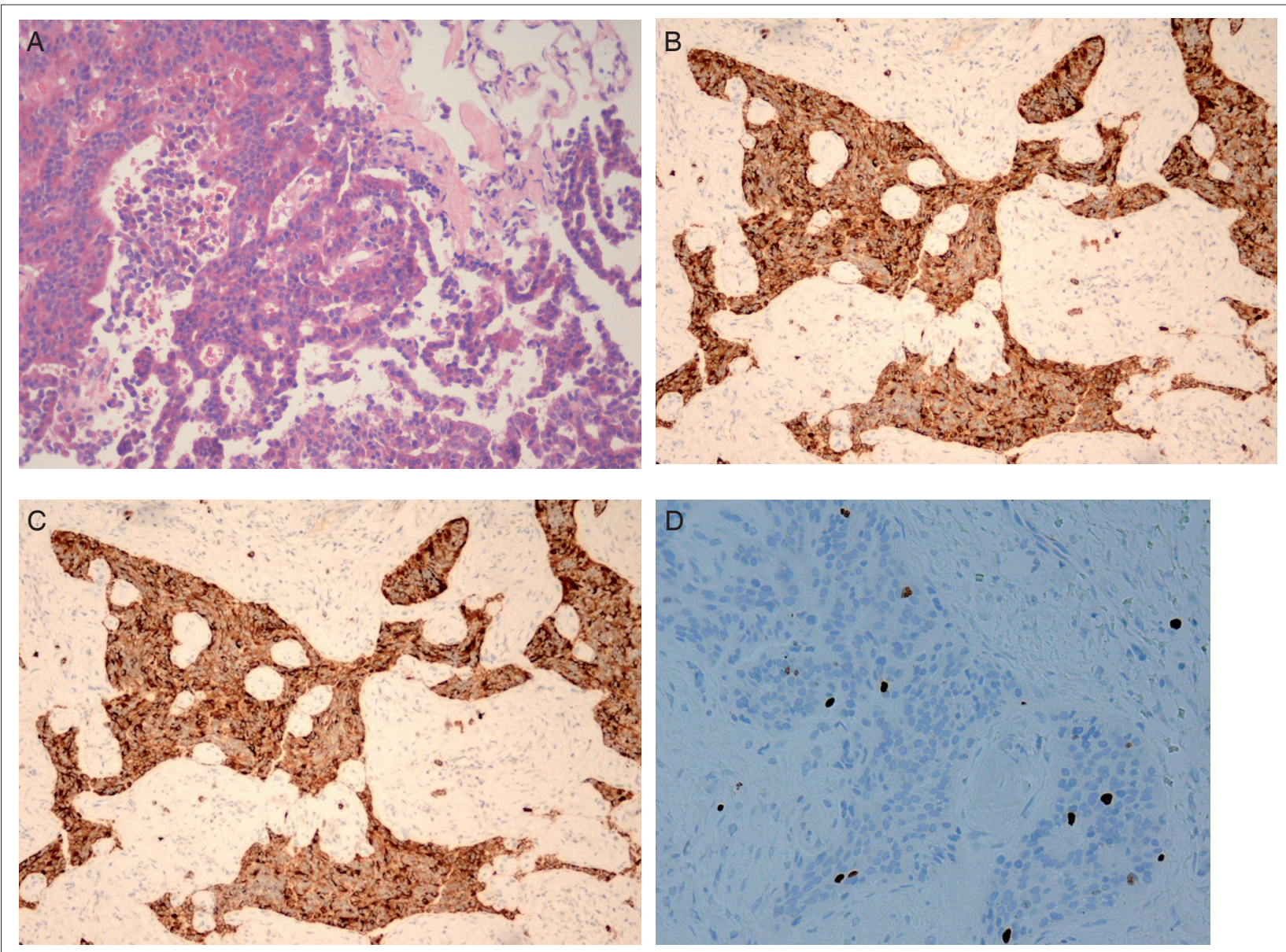

Figure 6. A: Well-differentiated papillary/trabecular/solid epithelial neoplasm composed of medium-sized cells. Central nuclei presented salt-and-pepper - like chromatin, and granular eosinophilic cytoplasm. Some neoplastic cells were seen amidst a fibrotic stroma. HE stain, original magnification x200. B-C: Immunohistochemical identification keratins marked by the antibodies $\mathrm{AE} 1+\mathrm{AE} 3$ and most of them were reactive for neuroendocrine markers chromogranin and synaptophysin. Original magnification x100. D: Ki-67 Ag was expressed in very low level ( $<1 \%$ of the cells). Original magnification $\mathrm{x} 400$.

\section{REVIEW OF THE LITERATURE}

In 1980, Murphy et al. first reported a middle ear adenoma with neuroendocrine differentiation, also called carcinoid tumor, as various denominations are used for these neoplasias, reflecting the controversy between its presumed histogenesis and differentiation (3). The lesion had adenomatous histological characteristics, but with ultrastructural neuroendocrine differentiation $(1,4)$. Since 
then, approximately 40 cases have been described in the English literature (1).

The incidence of this tumor in the middle ear is difficult to establish since it may be confused with adenomatous tumors, ceruminomas, and even with other tumors, such as paragangliomas (1).

Clinically, it presents with conductive hearing loss and ear fullness. Less frequently, patients may complain of tinnitus, headache, and even facial paralysis (5). In most cases, otoscopy demonstrates an intact tympanic membrane with a retrotympanic mass, as occurred in this case. Carcinoid syndrome (flushing, sweating, and diarrhea) is not found in these patients because of the small production of vasoactive peptides. Distant metastases are rare events $(1,5)$. Imaging studies consistently show a locally infiltrative mass and may show invasion of the ossicles (4).

Middle ear adenoma with neuroendocrine differentiation can be distinguished from adenomatous tumors (adenomas, adenocarcinomas, and adenoid cystic carcinomas), ceruminomas, and paragangliomas by their light microscopic appearance, argyrophilic granules, and ultrastructural evidence of neurosecretory granules, as well as by immunohistochemical detection of various neuroendocrine markers. These include neuron-specific enolase, chromogranin, synaptophysin, serotonin, glucagon, insulin, gastrin, vasoactive intestinal polypeptide, pancreatic polypeptide, and calcitonin (4).

\section{DISCUSSION}

We reported two cases of middle ear adenoma with neuroendocrine differentiation wich presented with unspecific clinical manifestations. In both cases, the radiological features were very similar to tympanic paraganglioma, wich is the most frequent tumoral lesion of the middle ear. The final diagnosis was provided by histological and immunohistochemical analysis of the tumor.

The recommended treatment is complete surgical excision of the lesion and the technique used is determined by the size and involvement of adjacent structures (1). Radiotherapy is not indicated since the tumor has poor radiosensitivity (1). The prognosis is good, however, a rigorous postoperative follow-up is indicated for all patients (3).

Our patients underwent surgical treatment and clinical follow-up in conjunction with the Oncology department. One of the patients (patient 1) had imaging studies performed 18 months after surgery, wich confirmed that there were no signs of disease recurrence.

\section{FINAL COMMENTS}

Middle ear adenoma with neuroendocrine differentiation is a rare entity, but it should be considered as a possible diagnosis in patients with hearing loss, tinnitus, aural fullness, and a retrotympanic mass.

\section{REFERENCES}

1. Ferlito A, Devaney K, Rinaldo A. Primary carcinoid tumor of the middle ear: A potentially metastasizing tumor. Acta Otolaryngol. 2006;126(3):228-31.

2. Pellini R, Ruggieri M, Pichi B, et. al. A case of cervical metastases from temporal bone carcinoid. Head Neck. 2005;27(7):644-47.

3. Becker CG, Barra IM, Pimenta HHO, Crosara PFTB, Ribeiro CA, Castro LPF. Adenoma da orelha média: relato de caso. Rev Bras Otorrinolaringol. 2004;70(4):551-4.

4. Knerer B, Matula C, Youssefzadeh S, et al. Treatment of a local recurrence of a carcinoid tumor of the middle ear by extended subtotal petrosectomy. Eur Arch Otorhinolaryngol. 1998;255(2):57-61.

5. Shibosawa E, Tsutsumi K, Ihara Y, et al. A case of carcinoid tumor of the middle ear. Ausis, Nasus, Larynx. 2003;30 Suppl:S99-S102. 